\title{
Prevalence, Pattern and Impact of Menstrual Ailments among Princess Nourah Bint Abdulrahman University Students in Riyadh, Saudi Arabia
}

\author{
Abyat N Alotaibi ${ }^{1}$, Aljawharah A Alkadi ${ }^{1}$, Aliah H Alanazi ${ }^{1}$, Ashwag A \\ Altilas $^{1}$ and Lamiaa A Fiala ${ }^{2,3 *}$ \\ ${ }^{1}$ Graduates, Epidemiology program, Department of Health Sciences, Faculty of \\ Health and Rehabilitation Sciences, Princess Nourah Bint Abdulrahman University, \\ Riyadh, Saudi Arabia \\ ${ }^{2}$ Department of Health Sciences, Faculty of Health and Rehabilitation Sciences, \\ Princess Nourah Bint Abdulrahman University, Riyadh, Saudi Arabia \\ ${ }^{3}$ Department of Public Health, Community, Environment and Occupational \\ Medicine, Faculty of Medicine, Suez Canal University, Egypt \\ *Corresponding Author: Lamiaa A Fiala, Department of Health Sciences, Faculty of \\ Health and Rehabilitation Sciences, Princess Nourah Bint Abdulrahman University, \\ Riyadh, Saudi Arabia and Department of Public Health, Community, Environment and \\ Occupational Medicine, Faculty of Medicine, Suez Canal University, Egypt.
}

\author{
Received: October 25,2021 \\ Published: November 19, 2021 \\ (C) All rights are reserved by Lamiaa A Fiala., \\ et al.
}

\begin{abstract}
Background: Menstruation is an essential change experienced by every female. The length of menstrual cycle varies from 21 to 35 days. Some females may experience problems that affect the normal cycle of menstruation referred to as menstrual ailments, which may range from heavy, painful periods to no periods at all. Among gynecological illnesses, the problems related to menstruation are the greatest common, affecting not only the female's quality of life but also their families, public and general economics. This study aims to determine the prevalence of and impact of menstrual ailments among Princess Nourah Bint Abdulrahman University students.

Methods: A cross-sectional study started in January 2021 to April 2021 among 518 female students. Multistage technique was used to recruit students from health and non-health colleges. Data designed to determine prevalence, pattern, and impact of menstrual ailments among Princess Nourah Bint Abdulrahman University.

Results: The results showed high prevalence of menstrual ailments among participant, the highest prevalence was pre-menstrual syndrome (28\%) and dysmenorrhea (25\%). According to the impact, 38\% of participants stated that they miss home activities, and $31 \%$ stated getting a sick leave due to menstrual ailments.

Keywords: Menstrual Cycle; Ailments; Female Students; Pattern; Prevalence; Impact; Princess Nourah Bint Abdulrahman University
\end{abstract}

\section{Introduction}

Every female experience natural changes in her teen years, physically and emotionally, which include the start of menstrual cycle [1]. In the years between puberty and menopause, menstruation happens. Menstruation is also referred to as "menses" or "period", which is every month blood flow from the uterus into the cervix and out through vagina. The average length of menstrual cycle is around 28 days, (normal range between 21 days and 35 days). The normal bleeding of nearly all women is about 3 to 5 days (average 2 to 7 days) [2].

Menstrual ailments/disorders are problems that affect the woman's normal menstrual cycle and have been defined as a disruptive physical and/or emotional symptoms just before and dur- 
ing menstruation, including heavy bleeding, missed periods and unmanageable mood swings [3].

There are various forms of menstrual problems, which may vary from heavy, serious, distressing menstruation cycles to no cycles at all. However, every woman should take in consideration whether her periods less than 21 days or over 3 months apart, or even if they last more than 10 days [2].

Examples of menstrual ailments or problems are menorrhagia which is the medical term for significantly heavier periods. Amenorrhea is absence of menstruation. Dysmenorrhea is severe, frequent cramping during menstruation. Oligo-menorrhea is a condition in which menstrual cycles are infrequent, occurring more than 35 days a part [4]. Premenstrual syndrome (PMS) is "a set of physical, emotional, and behavioral symptoms that occur a week before menstruation in most cycles" and usually the symptoms do not appear until around 13 days of the cycle and gone within 4 days after bleeding starts [2].

The problems related to menstruation are the greatest common gynecological illnesses. They do not affect females only but also influence their families, the public and the general economy too [5]. Menstruation can have a major impact on the quality of women's lives. Because of their menstruation, women felt that they are forced to abstain from social activities, got absent from work during the heaviest days of menstruation, have limitations in work and school and blocked from educational and academic achievements [6].

At the same time there are a lot of females that suffer from menstrual ailments/disorders that cause major problems to the female herself and her community. According to Saudi medical journal that published research studying the prevalence of menstrual problems in young female students, $91 \%$ of them were in pain from different menstrual ailments.

It is proved that their menstrual ailments did weaken their academic performance, increase their absenteeism, and absolutely cause a huge financial burden [7].

Study Objectives: To determine the prevalence, pattern and impact of menstrual ailments among Princess Nourah Bint Abdulrahman University students.

\section{Methodology}

This is a descriptive cross-sectional study design. It was conducted to determine the prevalence, pattern, and impact of menstrual ailments among Princess Nourah Bint Abdulrahman University students, Riyadh, Saudi Arabia. This study was conducted on students of Princess Nourah Bint Abdulrahman University, from January to April 2021. The total number of students in Princess Nourah Bint Abdulrahman University is 38986 [8]. All of Princess Nourah Bint Abdulrahman University students are included in the study except students who either removed their uterus or had amenorrhea. Sample size was calculated using the " $n^{4}$ studies application" software application [9], considering that the study is a cross-sectional one, and the target population is more than ten thousand, it gives a sample size of 518 students.

\section{Sampling technique}

colleges of Princess Nourah Bint Abdulrahman University are classified into four major fields (humanities' colleges, science colleges, community colleges, and health colleges). Each major field has different colleges, and every college has different academic programs. Multistage technique has been used to recruit the students from the colleges to represent the different major fields. First, colleges are stratified into health colleges and non- health colleges (humanities' colleges, science colleges, and community colleges). Then by simple random technique two colleges are selected: one college from the health colleges and another college from the non-health colleges. All students in the academic programs (clusters) are recruited in the study. Students was approached by online survey to distribute the study questionnaire, after making announcement on social media (using Twitter and WhatsApp applications).

The 516 sample students were divided proportionally between the two colleges according to the size of their students, as follows: $\mathrm{nh}=(\mathrm{Nh} / \mathrm{N}) * \mathrm{n}$ (where $\mathrm{nh}$ is the sample size for stratum $\mathrm{h}, \mathrm{Nh}$ is the population size for stratum $h, N$ is total population size, and $n$ is total sample size).

Population size of health colleges $=2476$ students, while population size of non-health colleges $=36510$ students. So, the planned sample size was 33 students recruited from health colleges, and 483 students was recruited from non-health colleges.

Data was collected using a pre-designed, self-administrated questionnaire including demographic data (3 questions), descrip-

Citation: Lamiaa A Fiala., et al. "Prevalence, Pattern and Impact of Menstrual Ailments among Princess Nourah Bint Abdulrahman University Students in Riyadh, Saudi Arabia". Acta Scientific Medical Sciences 5.12 (2021): 142-147. 
tion of the menstrual cycle (32 questions), and the impact of menstrual ailments (8 questions).

Menstrual problems are defined as follows [5]:

- Secondary amenorrhea: During the last three months there is no menstruation.

- Regular Period: The mensuration repeated once every twenty-eight to thirty-two days.

- Oligo-menorrhoea the menstruation is repeated every more than thirty-two days.

- Poly-menorrhoea: The menstruation is repeated every less than twenty-one days.

- Hypo-menorrhoea: The amount of blood loss less than 3 pads and the duration of the period equal or less than three days.

- Hyper-menorrhoea: The amount of blood loss more than five pads and the duration of the period equal or more than seven days.

- Dysmenorrhea: Pain during period, which is characterized 3 patterns mild, moderate or severe.

- $\quad$ Pre-menstrual Syndrome (PMS): Is a multi-symptom that happened ten days be- fore the starting of the menstruation, some of those symptoms such as bloating or swelling of the abdomen or painful or tender breasts, rapid mood change and, depressed mood. Having at least three of these symptoms in condition that those symptoms should vanish at the start of menstruation will consider as PMS.

The software JMP version 16 was used to analyses the collected data [10]. Data was revised, coded, and tabulated using the frequency and percentage.

\section{Ethical consideration}

participants were informed about the aim of the study, the voluntary participation in and withdrawal from the study, the anonymity of collected data was maintained, there is no private data was shared, and that their responses with was kept strictly confidential and used only for research purposes.

\section{Results}

This study was conducted on 518 students of Princess Nourah Bint Abdulrahman University. The population age ranged from 17

\begin{tabular}{|l|c|c|}
\hline Variable & N & \% of total \\
\hline Age & & \\
\hline 19 and less & 132 & $25.48 \%$ \\
\hline $20-23$ & 332 & $64.09 \%$ \\
\hline 24 and more & 54 & $10.42 \%$ \\
\hline Marital status & & \\
\hline Married or previously married & 57 & $11 \%$ \\
\hline Single & 461 & $89 \%$ \\
\hline Academic level & & \\
\hline First year & 163 & $31.47 \%$ \\
\hline Middle years & 169 & $32.63 \%$ \\
\hline Last year & 132 & $25.48 \%$ \\
\hline Internship & 54 & $10.42 \%$ \\
\hline
\end{tabular}

Table 1: Demographic Characteristic of the Study Sample

$$
(n=518) \text {. }
$$

to 24 , The study population age ranged from 17 to 24 years old. The majority of participants were between 20-23 years (332) and (461) were single while, the married were (57). According to academic level, (163) were in 1st year while, the minority of students (54) were in internship year (Table 1).

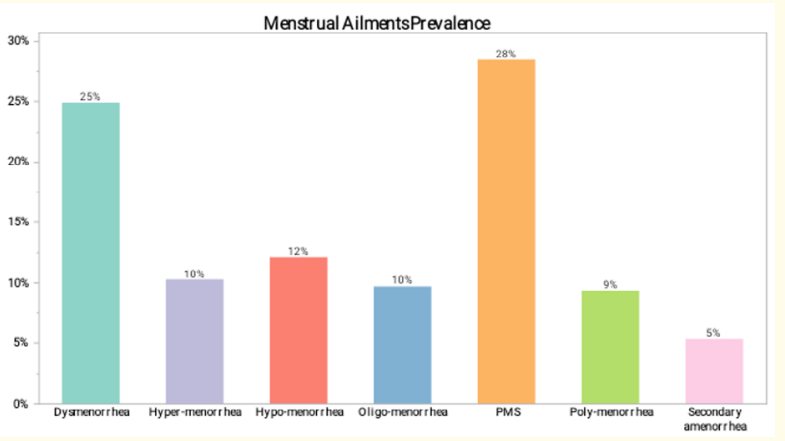

Figure 1: The Prevalence of Menstrual Ailments.

(Figure 1) shows the prevalence of menstrual ailments among the participants. The highest percentage of ailment among students were Pre-Menstrual Syndrome (PMS) with 28\%, near to it were Dysmenorrhea with 25\%. Hyper-menorrhea and oligo-menorrhea were the same with $10 \%$. Also, $12 \%$ and $9 \%$ of the participants had hypo-menorrhea and poly-menorrhea. While the minority had secondary amenorrhea with 5\%. 


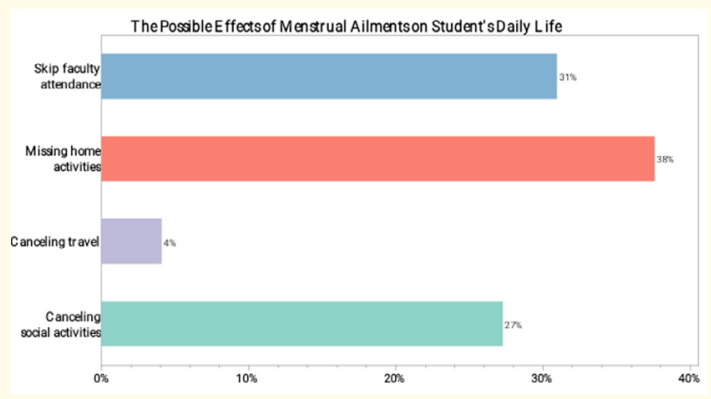

Figure 2: The Possible Effects of Menstrual Ailments on Student's Daily Life.

(Figure 2) illustrates the impact of menstrual ailments among study participants. Its apparent that missing home activities is the most frequent impact (38\%). Coming next to that is getting a sick leave $(31 \%)$. The least frequent impact among participants was canceling travel (4\%).

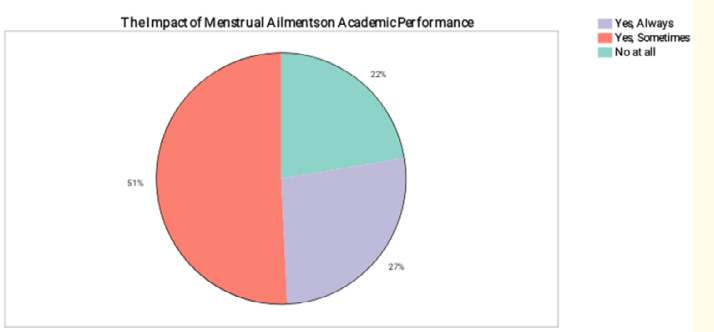

Figure 3: The Impact of Menstrual Ailments on Academic Performance.

(Figure 3) demonstrates If the menstrual ailments can have an effect on the academic performance or not. Almost half of the study participants stated that menstrual ailments affect their academic performance. On the other hand, $27 \%$ stated that it does not affect them at all.

\begin{tabular}{|c|c|c|}
\hline Variable & Frequency & Percent \% \\
\hline Age & & \\
\hline 19 or less & 97 & 18.73 \\
\hline $20-23$ & 239 & 46.13 \\
\hline 24 or more & 43 & 8.3 \\
\hline Marital status & & \\
\hline Married or previously married & 46 & 8.88 \\
\hline Single & 333 & 64.28 \\
\hline
\end{tabular}

Table 2: Prevalence and Pattern of Dysmenorrhea among Students $(\mathrm{n}=379)$.
(Table 2) shows the pattern of dysmenorrhea among the participants, the table stated that the highest percentage of dysmenorrhea among age groups was in participants aged 20-23, and in marital status $64 \%$ of the dysmenorrhea cases were in single participants while less than $10 \%$ in the married or previously married participants.

\begin{tabular}{|l|c|c|}
\hline Variable & Frequency & Percent \% \\
\hline Age & & \\
\hline 19 or less & 101 & 19.49 \\
\hline $20-23$ & 284 & 54.83 \\
\hline 24 or more & 48 & 9.26 \\
\hline Marital status & & \\
\hline Married or previously married & 52 & 10.03 \\
\hline Single & 381 & 73.55 \\
\hline
\end{tabular}

Table 3: Prevalence and Pattern of PMS among Students

$$
(n=433) \text {. }
$$

(Table 3) describe the patterns of premenstrual disorder among the participants. more than half of the participants suffer from PMS aged from 20 to 23 , which equals to 284 females, 101 females were 19 or less and 48 females 24 or more. $73 \%$ of the cases were in the single participants and only $10 \%$ married or previously married.

\section{Discussion}

Menstrual problems are the most common cause for women being referred to gynecologists, and these issues have a significant impact on women's lives and wellbeing. Menstrual issues have a certain level of privacy in some communities thus they do not find interest in the community [5].

In this study the results showed that a high percentage of student suffered from different kind of menstrual ailments; $9 \%$ of students suffered from poly-menorrhea. In a study in Lebanon Around half of the participants (51.4\%) reported polymorphous [5]. This is concerning as previous research has shown that menstrual blood loss has a negative impact on women's quality of life and that excessive bleeding may put women at risk of anemia [5]. This study shows prevalence of oligomenorrhoea with $10 \%$. Previous study sites in five counties in rural Missouri and the city of St. Louis conducted that oligomenorrhoea is a factor that raises the risk of psychological diseases such as anxiety [11]. Similarly, other study in China demonstrates that younger women are more likely to suffer from oligomenorrhea. Moreover, women with oligomenorrhea had a higher prevalence of family history of oligomenorrhea and 
were exposed to stressful conditions [8]. According to other study, women with high PMS reported significantly higher stress and lower quality of life [12], this finding explains the high percentage of PMS in this study among the students with $28 \%$ which is known that students suffer from high study stress. This study showed that $12 \%, 10 \%$ had respectively hypomenorrhea and hypermenorrhea, this may be due to changing trends in lifestyle, dietary habit, stress, hormonal imbalance or some medical reasons which requires gynecological assessment at the earliest [5].

This study is showing how the menstrual ailments can affect the participants' daily life. A study conducted among 40,000 Dutch females $34 \%$ of the participants stated that their daily activities were interrupted by premenstrual symptoms, $22 \%$ of them had to reduce their usual work and $11 \%$ had to delegate their tasks [13]. This study mentions more details about the types of activities affected by the menstrual symptoms. The highest percentage (38\%) had to Avoid their home activities which is close to the percentage in the Dutch study mentioned previously, and another third getting a sick leave from their college attendance and social engagements. The percentages may differ between this study and the Dutch study which may be due to the difference in the distribution of the ailments and the age of the participants. But it still considered a large portion of the participants had to cancel their home activities, college classes and social engagements affecting their social life and academic performance [13].

As regard to the impact menstrual ailments leave on student's life, the majority of the participants agreed that these disorders can cause a clearly seen effect on them. Participants from different academic levels were asked if menstrual ailments affect their academic performance or not, great number of females chose yes for this question. however, students in the middle years, which represent levels three, four, five and six got the highest percentages. In conformity with a study that was carried in Princess Nourah Bint Abdulrahman University in Riyadh about the impact of menstrual periods on performance of female workers, study sample was taken from different departments and represents different ages to insure variety and representativeness, although study started with a hypothesis that menstruation didn't have any impact on the work performance, the result showed the complete opposite, study sample results were divided into two sections, The greater part of the participants explained that their work was less professional throughout the time of menstruation, while the rest of the study sample admitted that they work differently at that specific time [9]. this might be attributed to the high levels of pain and symptoms expressed by the participants during assessment.

In this study, the prevalence of dysmenorrhea was higher among students aged $20-23$ with $46.13 \%$, previous study conducted in Turkey indicated that the students' prevalence of dysmenorrhea was found to be $72.7 \%$, a reason for the variation in these estimates may be the use of selected groups of women and the lack of a widely recognized method of identifying dysmenorrhea, which may be the one explanation for the disparity which is likely as much to blame as the data collection techniques, research meanings of dysmenorrhea and pain, and the study populations themselves [14]. Similar to this study, many studies determined that the prevalence of dysmenorrhea showed a decrease with increasing age [14]. Also, other studies indicated that there are no significant associations were detected between marital status and dysmenorrhea $[15,16]$.

About premenstrual syndrome (PMS) and its prevalence and pattern, participants displayed high percentage of females going through PMS, it was according to two variables, age and marital status. Females with ages 20-23 took the highest percentage compared to other ages of older and younger. this might be attributed to the fact the premenstrual syndrome PMS is more frequent in females during their reproductive years, according to the National Center for Biotechnology Information that conducted a study about Epidemiology of Premenstrual Syndrome (PMS) [1], and if the female was single this increased the prevalence by $73.55 \%$ which represents almost all of the study sample. In agreement with a study conducted in India to compare and assess premenstrual symptoms between married and single females, their results revealed that unmarried women experienced more premenstrual symptoms than married women [2].

\section{Conclusion and Recommendation}

This research has revealed that there is a high proportion of students suffering from menstrual ailments. These ailments can influence the female's quality of life and her general life satisfaction.

Initiation of campaigns, spreading brochures and including courses in the school's educational plans can help early recognition and treatment of menstrual ailments. 


\section{Limitations}

The duration of the study was relatively short, which was not helpful in all phases of the study.

\section{Bibliography}

1. "Menstrual Cycle". Ministry of Health Saudi Arabia (2020).

2. "Menstrual disorders Information"| Mount Sinai - New York. Mount Sinai Health System (2020).

3. "Overview". Healthy Women (2020).

4. "Abnormal Menstruation (Periods): Types, Causes and Treatment" (2020).

5. Karout N. "Prevalence and pattern of menstrual problems and relationship with some factors among Saudi nursing students". Journal of Nursing Education and Practice 5.12 (2015): 1-5.

6. Kadir RA., et al. "The impact of menstrual disorders on quality of life in women with inherited bleeding disorders". Haemophilia: The Official Journal of the World Federation of Hemophilia 16.5 (2010): 834.

7. Rafique N and Al-Sheikh MH. "Prevalence of menstrual problems and their association with psychological stress in young female students studying health sciences" 39.1 (2018): 67-73.

8. Princess Nourah Bint Abdul Rahman University. Riyadh, Saudi Arabia.

9. Ngamjarus $\mathrm{C}$ and Chongsuvivatwon V. "n4Studies". The Royal Golden Jubilee Ph.D. Program - The Thailand Research Fund, prince of Songkla University (2013).

10. New in JMP 16. Jmp.com. (2021).

11. Barron M., et al. "Associations Between Psychiatric Disorders and Menstrual Cycle Characteristics". Archives of Psychiatric Nursing 22.5 (2008): 254-265.

12. Lustyk M., et al. "Stress, Quality of Life and Physical Activity in Women with Varying Degrees of Premenstrual Symptomatology". Women and Health 39.3 (2004): 35-44.

13. Hantsoo, L. "The impact of Menstrual symptoms on everyday life: A survey AMONG 42,879 Women". (2021).

14. Unsal A., et al. "Prevalence of dysmenorrhea and its effect on quality of life among a group of female university students". Upsala Journal of Medical Sciences 115.2 (2010): 138-145.
15. Ju H., et al. "The Prevalence and Risk Factors of Dysmenorrhea”. Epidemiologic Reviews 36.1 (2013): 104-113.

16. JAMIESON D and STEEGE J. "The Prevalence of Dysmenorrhea, Dyspareunia, Pelvic Pain, and irritable bowel syndrome in Primary Care Practices". Obstetrics and Gynecology 87.1 (1996): 55-58.

Volume 5 Issue 12 December 2021

(c) All rights are reserved by Lamiaa A Fiala., et al.

Citation: Lamiaa A Fiala., et al. "Prevalence, Pattern and Impact of Menstrual Ailments among Princess Nourah Bint Abdulrahman University Students in Riyadh, Saudi Arabia". Acta Scientific Medical Sciences 5.12 (2021): 142-147. 